\title{
O conhecimento dos agentes comunitários de saúde acerca da assistência em saúde mental no município de Macapá, Amapá
}

\section{The knowledge of community health agents about mental health care in the municipality of Macapá, Amapá}

\author{
Blenda Matos da Silva ${ }^{1^{*}}$, Maria Raquel da Silva de Oliveira ${ }^{2}$, Washington Luiz de Oliveira \\ Brandão ${ }^{3}$
}

${ }^{1}$ Graduada em psicologia Instituto Macapaense de Ensino Superior-IMMES, Macapá, Amapá, Brasil. https://orcid.org/0000-0002-4540-3220 E-mail: blendamatoa@gmail.com *Autor de correspondência

${ }^{2}$ Graduada em Psicologia, Instituto Macapaense do Melhor Ensino Superior, Macapá-AP Brasil. https://orcid.org/0000-0003-4797-0576 Email: raquelanjod89@gmail.com

${ }^{3}$ Psicólogo, Doutor em Psicologia (Teoria e Pesquisa do Comportamento), professor magistério superior da Universidade Federal do Amapá. Macapá-AP Brasil. https://orcid.org/0000-0001-9800-9991 E-mail: brandao@unifap.br

\author{
Palavras-chave \\ Agente Comunitário de Saúde e \\ Saúde Mental \\ Assistência em Saúde Mental \\ Saúde Mental na Atenção Básica
}

\section{Keywords}

Community Health Agent and Mental Health

Mental Health Care. Primary care ACS Mental health
O objetivo desta pesquisa foi analisar o conhecimento que os agentes comunitários de saúde (ACS's) apresentam acerca da assistência em saúde mental no município de Macapá-AP. Trata-se de uma pesquisa exploratóriodescritiva de natureza qualitativa. Participaram deste estudo 6 Agentes Comunitários de saúde de duas equipes que atuam no Estratégia Saúde da Família, lotados na Unidade Básica de Saúde Dr. Lélio Silva, localizada na cidade de Macapá, capital do Estado do Amapá. A coleta de dados foi realizada através de uma entrevista semiestrurada composta de dez perguntas direcionadas aos Agentes Comunitários de Saúde. Os resultados deste estudo mostraram que a maioria dos ACS consegue definir adequadamente seu papel na atenção básica. Foi possível notar que este, por estar engajado no território, tem um potencial de escuta que facilita o desenvolvimento de ações voltadas para demandas específicas, porém, muitas vezes acabam reproduzindo o modelo biomédico, centrado apenas no tratamento e na medicalização de doenças, sem promover ações voltadas para a prevenção no campo da saúde mental e isso acaba divergindo com o modelo de cuidado pensado pela ESF e dispositivos de cuidado em saúde mental. Foi perceptível também a falta de comunicação e articulação internamente na atenção básica e na Rede de Saúde Mental, bem como, a ausência de apoio matricial que proporcionaria romper com a lógica do encaminhamento, muitas vezes vinculada a lógica da desresponsabilização e também contribuiria para a facilitação do processo de trabalho.

The objective of this research was to analyze the knowledge that community health agents (CHAs) have about mental health care in the city of Macapá-AP. This is an exploratory-descriptive research of qualitative nature. Six Community Health Agents of two teams that work in the Family Health Strategy, based in the Dr. Lélio Silva Basic Health Unit, located in the city of Macapá, capital of the state of Amapá, participated in this study. Data collection was performed through a semi-structured interview directed to Community Health Agents. The results of this study showed that most ACS can define their role as disease prevention, information gathering, demand identification and referral, as well as promoting health. Being engaged in the territory has a listening potential that facilitates the development of actions focused on specific demands, however, these professionals often end up reproducing the biomedical model, centered only on the treatment and medicalization of diseases, without promoting actions focused on prevention in the field of mental health and this ends up diverging with the care model thought by the FHS and mental health care devices. It was also noticeable the lack of communication and articulation of the devices of the mental health network, as well as the lack of matrix support that would break with the logic of referral, often linked to the logic of lack of responsibility and also contribute to the facilitation of the work process.

\section{INTRODUÇÃO}

Depois da criação do Sistema Único de Saúde (SUS), surgiram os Agentes Comunitários de Saúde (ACS) que foram incluídos nesse sistema no início da década de 1990. Após isso, em 1994, assomou também o Programa Saúde da Família (PSF) que teve como objetivo melhorar a saúde e a ordenação da Atenção Básica. As atividades básicas do agente comunitário de saúde são prevenção de doenças e promoção de saúde por meio de ações educativas, e com a criação do PSF esse modelo de organização passou a ser centrado na família com ênfase territorial e trabalho em equipe.

Com a Reforma Psiquiátrica foi implantado um novo modelo de cuidado em saúde mental, por uma rede integrada de atenção através da Lei Federal no 10.216/2011 
(BRASIL, 2011a) que dispõe sobre a proteção e os direitos das pessoas com transtornos mentais, redireciona a assistência em saúde mental e privilegia o oferecimento de tratamento em serviço de base comunitária. Deste modo, o ACS ganha um importante papel, não somente na Atenção Básica, mas agora também no cuidado em Saúde Mental.

O Decreto $\mathrm{n}$ - 7.508/2011 estabeleceu portas de entrada no cuidado em saúde mental no SUS e a portaria 336/2002 regulamentou os Centros de Atenção Psicossocial (CAPS) (BRASIL, 2011b). Os CAPS são núcleos de atendimentos às necessidades de saúde mental, que incluem o usuário às redes de apoio no seu próprio território e preservam a sua cidadania (BRASIL, 2002).

A Reforma Psiquiátrica e a ESF tem princípios que convergem: romper com o modelo hospitalocêntrico de assistência, buscar a participação da família no tratamento, promover a participação comunitária e buscar a melhoria da qualidade de vida das pessoas, bem como, fazem parte da Rede Atenção Psicossocial (RAPS).

A RAPS foi destinada para estruturar as ações de saúde mental juntamente com a atenção básica, como a Estratégia Saúde da Família (ESF), ambulatórios, leitos de internação em hospitais gerais e ações de suporte e reabilitação psicossocial (QUINDERÉ; JORGE; FRANCO, 2014).

Depois de todas as modificações feitas no sistema de saúde, o ACS assumiu um importante papel neste cenário de promoção de saúde. Segundo Fortes e Spinetti (2004), o ACS exerce um papel de "elo" entre a equipe e a comunidade, já que este é o profissional que tem primeiro contato com a comunidade. Além disso, identifica problemas, orienta, encaminha e acompanha a realização de procedimentos necessários à prevenção de doenças, promoção de saúde e a recuperação/reabilitação da saúde das pessoas da comunidade (CARDOSO; REINALDO; CAMPOS, 2008).

Porém, apesar de toda a articulação gerada pelos avanços advindos da Reforma Psiquiátrica e levando em consideração o ACS como operador central da ESF, estudos de diversos autores como: SILVEIRA E VIEIRA, (2009); CORREIA; BARROS; COLVEIRO, (2011); WADMAN, COSTA; PAIANO, (2012); SILVA et al, (2014); SILVA-JUNIOR et al, (2016); VIEIRA et al (2017); SILVA, NÓBREGA; OLIVEIRA (2018), demostraram que na prática, os ACS's tem a necessidade de capacitação acerca da saúde mental.

Nesse contexto, pode-se observar que o ACS se depara constantemente com problemas de saúde mental em seu trabalho, entretanto, este não está capacitado a desenvolver as ações de saúde mental atribuídas à atenção básica, visto que, desconhecem sinais/sintomas da doença, formas de tratamento alternativos e complementares, serviço de apoio, e não receberam nenhuma capacitação sobre o tema. É notória a importância da capacitação em saúde mental para esses profissionais, pois poderá levar a maior adesão dos indivíduos a rede de atenção básica e melhor articulação com outros serviços de atenção (CARDOSO; REINALDO; CAMPOS, 2008).

O fortalecimento da rede de assistência em saúde mental pode estar vinculado ao conhecimento e, consequentemente com o envolvimento que os técnicos da rede de atenção psicossocial tem com seus respectivos papéis no processo de cuidado. Neste contexto, é importante analisar qual o conhecimento que os agentes comunitários de saúde tem sobre a assistência a saúde mental e assim identificar os potenciais aspectos para o fortalecimento da rede de atenção psicossocial.

Este estudo torna-se importante para a consolidação de práticas voltadas para um cuidado adequado as pessoas, possibilitando a integração de forma positiva dos dispositivos de assistência em saúde mental, tanto no fortalecimento da necessidade de formação continuada, quanto no fazer diário em busca de um cuidado mais humanizado.

Sendo assim, o objetivo geral desta pesquisa é analisar o conhecimento que os Agentes Comunitários de Saúde (ACS's) apresentam acerca da assistência em saúde mental e comportamento suicida no município de Macapá-AP. Os objetivos específicos são: Identificar as atribuições dos ACS's no campo da promoção de saúde mental; Caracterizar a compreensão que os ACS's têm acerca da assistência em saúde mental no município de Macapá-AP; Compreender o conhecimento que os ACS's têm acerca da assistência ao comportamento suicida.

\section{A reforma psiquiátrica}

O processo de Reforma Psiquiátrica eclodiu no Brasil, paralelo ao "Movimento Sanitário" na década de 70 e esteve atrelado ao contexto internacional de mudanças pela superação de violência asilar. Tais movimentos reivindicavam mudanças nos modelos de atenção e gestão em saúde. Apesar de paralelos, o processo de Reforma Psiquiátrica foi um movimento singular que se iniciou com o Movimento dos Trabalhadores em Saúde Mental (MTSM) em 1978, formado por trabalhadores integrantes do movimento sanitário, associações de familiares, sindicalistas, membros de associações de profissionais e pessoas com longo histórico de internações psiquiátricas. Estes denunciavam a violência nos manicômios, a hegemonia de uma rede privada de assistência, e coletivamente criticaram o chamado saber psiquiátrico e o modelo hospitalocêntrico na assistência às pessoas com transtornos mentais (AMARANTE; GIOVANELLA, 1998).

A Reforma Psiquiátrica trata-se de um processo político e social complexo, para além das mudanças na legislação. 
Compreende-se como um conjunto de transformações de práticas, saberes, valores culturais e sociais, e é no cotidiano da vida das instituições, dos serviços e das relações interpessoais que o processo da reforma avança, marcado por dificuldades, tensões, conflitos e desafios (NUNES, 2018).

Após o surgimento do Primeiro Centro de atenção Psicossocial (CAPS) que estabeleceu novos modelos de cuidado em saúde mental no Brasil, do processo de intervenção da Secretaria Municipal de Saúde de Santos no Hospital Psiquiátrico Anchieta que denunciou maus-tratos e mortes de pacientes, da implantação de Núcleos de Atenção Psicossocial e residências terapêuticas para egressos do hospital e associações, foi que em 1989 dá entrada no congresso Nacional o projeto de lei do Deputado Paulo Delgado (PT/MG), que propõe a regulamentação dos direitos das pessoas com transtornos mentais e a extinção progressiva dos manicômios no país, representando um enorme avanço da Reforma Psiquiátrica Brasileira no campo normativo (BRASIL, 2005).

Contudo, os direitos das pessoas com transtornos mentais e as práticas em saúde mental só foram regulamentadas através da Lei Federal 10.216 de 2001, conhecida como Lei da Reforma Psiquiátrica e da Portaria 336 de 2002, que organizou os Centros de Atenção Psicossociais (CAPS) (BRASIL, 2005).

Com a promulgação da lei 10.216 e a realização da III Conferência Nacional de Saúde Mental, que a política de saúde mental do governo federal, junto com as diretrizes da Reforma Psiquiátrica afirmaram-se, ganhando maior sustentação e visibilidade. Neste cenário, foi criado o Programa "De volta para casa", como impulso a desinstitucionalização de pessoas com longo histórico de internação. Como complemento, foi criada também uma política de recursos humanos para a Reforma Psiquiátrica, a política para a questão do álcool e outras drogas, incorporando a estratégia de redução de danos (TENÓRIO, 2002).

Os CAPS, segundo o Ministério da Saúde, foram criados como serviços substitutivos às internações em hospitais psiquiátricos e são responsáveis também pela articulação de ações em saúde mental de um município, visando atender pessoas com transtorno mental (BRASIL, 2004a).

Os Centros de Atenção Psicossocial (CAPS), detém um papel estratégico na articulação da Rede e na construção da política de saúde mental. Cabe ao CAPS oferecer o acompanhamento clínico e possibilitar a "reinserção social" dos usuários no trabalho, na comunidade e na família, por meio de práticas intersetoriais (sócio sanitárias, jurídicas, cooperativas de trabalho, escolas, empresas). Além disso, o CAPS deve dar suporte à saúde mental na rede básica através do trabalho conjunto com as equipes de saúde da família (SCHNEIDER et al., 2014).

De acordo com Vieira et al. (2017), com os avanços, a Política de Saúde mental e Política de Humanização foram implementadas a ESF e as Redes de Atenção em Saúde, pelo Decreto n.7.508/2011 que regulamentou a Lei Orgânica da Saúde (Lei 8.080/90) no que diz respeito à organização do SUS, ao planejamento da Saúde, à assistência à saúde e à articulação interfederativa e definiu a rede como um conjunto de ações e serviços de saúde estruturados em níveis de complexidade sucessiva, com a finalidade de garantir a integralidade da assistência à saúde" (item VI, art 2ㅇ).

Deste modo, instituiu-se uma rede com várias portas de entrada no sistema, onde a atenção básica é uma delas, mas há também os hospitais de emergências, os CAPS, as residências terapêuticas, os hospitais gerais e outros que podem ser encontrados pelos próprios usuários na busca pelo cuidado (CECÍLIO, 1997).

\section{A rede de atenção psicossocial}

A Portaria 3.088/2011 criou a Rede de Atenção Psicossocial (RAPS) e definiu o modelo de atenção à saúde mental com foco na atenção comunitária, participação social e ênfase na atenção ao usuário de crack, álcool e outras drogas, com um modelo de gestão regional. Os objetivos dessas diretrizes contemplam o aumento do acesso do usuário através de diversas portas de entrada e o foco no usuário de substâncias psicoativas (TRAPÉ; CAMPOS, 2017).

Segundo a Portaria Ministerial 3088/2011, os objetivos gerais da Rede de Atenção Psicossocial são: ampliar o acesso à atenção psicossocial da população em geral, promover o acesso das pessoas com transtornos mentais e com necessidades decorrentes do uso de crack, álcool e outras drogas e suas famílias aos pontos de atenção e garantir a articulação e integração dos pontos de atenção das redes de saúde no território, qualificando o cuidado por meio do acolhimento, do acompanhamento contínuo e da atenção às urgências (BRASIL, 2011).

A Portaria supracitada traz também sobre os pontos de acesso que constituem a RAPS, são eles:

\section{I - Atenção Básica em Saúde;}

- Unidade Básica de Saúde (UBS);

- Núcleo de Apoio a Saúde da Família;

- Consultório na Rua;

- Apoio aos Serviços do componente Atenção Residencial de Caráter Transitório;

- Centros de Convivência e Cultura.

II - Atenção Psicossocial Especializada;

- Centros de Atenção Psicossocial, nas suas diferentes 
modalidades.

\section{III - Atenção de Urgência e Emergência;}

- SAMU 192;

- Sala de Estabilização;

- UPA 24 horas e portas hospitalares de atenção à urgência/pronto socorro, Unidades Básicas de Saúde.

IV - Atenção Residencial em caráter transitório;

- Unidade de Acolhimento;

- Serviço de Atenção em Regime Residencial.

V - Atenção Hospitalar;

- Enfermaria especializada em Hospital Geral;

- Serviço Hospitalar de Referência para Atenção às pessoas com sofrimento ou transtorno mental e com necessidades decorrentes do uso de crack, álcool e outras drogas.

VI - Estratégias de desinstitucionalização;

- Serviços Residenciais Terapêuticos;

- Programa de Volta para Casa VII - Reabilitação Profissional;

- Iniciativas de Geração de Trabalho e Renda;

- Empreendimentos Solidários e Cooperativas Sociais.

Os dispositivos devem estar interligados entre si, visando impedir a desintegração dos atendimentos, bem como, devem estar harmonizados objetivando a facilitação da elaboração do plano de ações nos determinados territórios, essencialmente no que diz respeito as visitas domiciliares e às intervenções clínicas na rede de suporte social e de relações pessoais significativas para o utente do serviço (VIEIRA FILHO; NÓBREGA, 2004).

Os autores Quinderé, Jorge e Franco (2014) conceituam a rede como:

(...) um tipo de rede que se constitui sem modelo, que não parte de uma estrutura, pois se constrói em ato, com base no trabalho vivo de cada trabalhador e equipe, mediante fluxos de conexões entre si, na busca do cuidado em saúde, seja em encaminhamentos realizados, procedimentos partilhados, projetos terapêuticos que procuram consistência no trabalho multiprofissional. (p.255)

Dessa forma, foi possível perceber a instauração de uma rede com vários dispositivos de entrada que devem estar articulados entre si, sem modelo padrão, mas com vistas a se adequar às necessidades de cada usuário, onde cada trabalhador é ator neste cenário.

\section{O agente comunitário de saúde}

Os agentes comunitários de saúde foram agregados ao
SUS em 1991, com a criação do Programa de Agentes Comunitários de Saúde (PACS), contudo, a profissão só foi regulamentada pela Lei N.10.507/2002, que definiu suas funções básicas: atividades de prevenção de doenças e promoção de saúde via ações educativas (VIEIRA et al., 2017).

Mais tarde, em 1994, o Ministério da Saúde instituiu a Estratégia de Saúde da família no Brasil (ESF). Onde o ACS passou a integrar uma equipe multidisciplinar, ocupando um lugar de destaque nas ações de atenção primária à saúde da comunidade. Normalmente eles possuem o ensino médio e na maioria dos casos tem conhecimento limitado sobre as doenças trabalhadas. Realiza atendimentos domiciliares relacionados à saúde: detecta problemas, orienta, encaminha e acompanha a realização dos procedimentos necessários para as pessoas da comunidade. São vistos como operadores centrais da ESF, sendo de grande importância para o bom andamento das ações de saúde realizadas pela equipe, bem como as ações voltadas para saúde mental (CONSOLI; HIRDES; COSTA, 2004).

As ações da atenção básica que são desenvolvidas pelo Programa de Saúde da Família, contam com uma equipe composta por médico, enfermeiro, técnico de enfermagem, agentes comunitários de saúde, entre outros profissionais responsáveis pelo atendimento à comunidade, estes também contam com o Núcleo de Apoio a Saúde da Família (NASF-AB) que se trata de uma equipe interdisciplinar formada por profissionais de várias áreas. $\mathrm{E}$ a partir disso, o trabalho do ACS se torna fundamental, pois desempenham a tarefa de identificar dificuldades de vida das pessoas, riscos, vulnerabilidades e doenças presentes nas famílias para, então, direcioná-los a equipe do PSF (LOPES; SOUZA, 2010).

A Política Nacional da Atenção Básica (PNAB), atualizada em 2017, traz as atribuições dos profissionais da Atenção Básica, entre eles, o ACS, e são atribuições destes: desempenhar ações que visem a promoção da saúde, de prevenção de doenças e agravos, e de vigilância em saúde, através de visitas domiciliares frequentes e de ações educativas individuais e coletivas, na UBS, no domicílio e outros lugares da comunidade, bem como a investigação epidemiológica de casos suspeitos de doenças e agravos junto a outros profissionais da equipe, se necessário; fazer visitas domiciliares continuadamente de acordo com o planejamento da equipe e as necessidades de saúde da população, para o acompanhamento das famílias e indivíduos do território, essencialmente as famílias com maior vulnerabilidade; reconhecer e registrar situações que alterem o curso das doenças ou que sejam relevantes para a epidemiologia relacionada aos fatores ambientais, realizando, quando necessário, o impedimento da propagação de doenças infecciosas e agravos; informar a 
comunidade acerca de sintomas, riscos e agentes transmissores de doenças e formas de prevenção individual e coletiva; realizar o encaminhamento dos usuários para a unidade de saúde de referência, registrar e comunicar o fato à autoridade de saúde responsável pelo território; entender e orientar as pessoas quanto à utilização dos serviços de saúde disponíveis; incentivar a comunidade a participar das políticas públicas voltadas para a saúde; identificar parceiros e recursos na comunidade que possam potencializar ações intersetoriais de relevância para a promoção da qualidade de vida da população, como ações e programas de educação, esporte e lazer, assistência social, entre outros.

Uma das principais atribuições do ACS na ESF é coletar informações referentes às necessidades de saúde da população, identificar usuários com problemas e encaminhálos a unidade de saúde. E por ser integrante da comunidade, o ACS torna-se um mediador e facilitador na relação entre o serviço de saúde e seu usuário, estabelecendo uma comunicação interpessoal efetiva, pois as pessoas por compartilharem da mesma realidade local, identificam-se com esse agente (WAIDMAN; COSTA; PAIANO, 2012).

O ACS presta acompanhamento aos pacientes em sua área de atuação e serve de ligação entre os usuários e o serviço de saúde, bem como transita entre o equipamento representante do saber biomédico e convive com os munícipes, o que nos mostra de forma significativa a sua importância, levando em consideração a sua posição que facilita a assistência (NUNES et al., 2002)

Segundo Barbosa e Dantas (2013), o papel do ACS é trabalhar para melhoria de vida e condições de saúde da sociedade, essencialmente em relação à mudanças de hábitos que melhorem a qualidade de vida, tendo como ferramenta de trabalho a educação em saúde. Ser intermediador entre o serviço de saúde e a comunidade é um papel de grande relevância.

Devido a grande mudança no cenário do modelo de saúde no Brasil, ressalta-se o papel de destaque que o agente comunitário de saúde (ACS) ganhou. Segundo Cardoso, Reinaldo e Campos (2008), o ACS tem um papel relevante, devido ao vínculo que ele possui com a comunidade adstrita; como membro dessa população tem uma maior facilidade de interagir com os mesmos e facilitar a integração da população com os serviços de saúde.

Os ACS estão em contato direto com a população e são os que mais identificam problemas de saúde a serem trabalhados pela equipe. Dentre os possíveis problemas estão às manifestações de alterações de comportamento de pessoas com transtornos mentais, que por sua vez, devido à reforma psiquiátrica e ao processo de desinstitucionalização estão retornando ao convívio em sociedade e às suas famílias (CARDOSO; REINALDO; CAMPOS, 2008).
De acordo com Cabral e Albuquerque (2015), o ACS é o membro da ESF de maior proximidade com a realidade local e vinculação com os usuários, que funciona como elo entre comunitários e ESF, é um parceiro potencial para as estratégias de cuidado em saúde, em especial na área de saúde mental, pois reside no território, experimentam situações similares as vividas pelos usuários, detecta os casos e passa as informações para a equipe. Desempenha também o papel de tradutor de orientações técnicas ao propiciar uma linguagem acessível à população (SOUZA, 2007; PEREIRA, 2007 apud CABRAL e ALBUQUERQUE, 2015, p. 160).

A conjectura do atendimento em rede se faz necessário para que o ACS possa colaborar com a construção de outros encaminhamentos para ampliar o atendimento ás pessoas com transtornos mentais, considerando que o oferecimento de ações em saúde mental não é atividade exclusiva dos CAPS, este deve estar articulado com a atenção básica, residências terapêuticas, ambulatórios, centros de convivência e clubes de lazer (LOPES; SOUZA, 2010).

\section{A importância do matriciamento na assistência em saúde mental}

Matriciamento, que no campo da saúde, é uma palavra que indica a possibilidade de sugerir que profissionais de referência e especialistas mantenham uma relação horizontal e não vertical, ou seja, sugerindo a interdisciplinaridade para discutir quais os benefícios e dificuldades para sua aplicação. Este processo de integração da saúde mental à atenção primária na realidade brasileira tem o norteador das experiências implementadas em diversos municípios, ao longo dos últimos anos, conhecido como apoio matricial, formulado por Campos (1999), tem estruturado em nosso país um tipo de cuidado colaborativo entre a saúde mental e a atenção primária.

O Matriciamento é um suporte desenvolvido para mobilizar profissionais de diversas áreas e especialistas diferentes para formar uma equipe interdisciplinar com o intuito de ampliar o campo de atuação e qualificar suas ações. "Sendo uma proposta de apoio para unir duas ou mais equipes, para um processo de construção de conhecimento compartilhada, criando propostas de intervenção pedagógico-terapêutica" (MINISTÉRIO DA SAÚDE, 2011, p. 13).

"O apoio matricial tem possibilitado, no Brasil, um cuidado colaborativo entre a saúde mental e a atenção primária" (MINISTÉRIO DA SAÚDE, 2011 p. 13), e essa relação amplia a possibilidade de realizar a clínica ampliada e a integração e diálogo entre diferentes especialidades e profissões (CAMPOS; DOMITTI, 2007 apud MINISTÉRIO DA 
SAÚDE, 2011).

Segundo Campos e Domitti (2007, p. 400), a relação entre essas duas equipes constitui um novo arranjo do sistema de saúde: apoio matricial e equipe de referência são, ao mesmo tempo, arranjos organizacionais e uma metodologia para gestão do trabalho em saúde, objetivando ampliar as possibilidades de realizar-se clínica ampliada e integração dialógica entre distintas especialidades e profissões.

Os instrumentos utilizados pelos profissionais para a realização do matriciamento incluem a elaboração do projeto terapêutico singular no apoio matricial de saúde mental, a interconsulta, a visita domiciliar conjunta, o contato à distância, o genograma, o ecomapa, a educação permanente em saúde mental e a criação de grupos na atenção primária à saúde (CHIAVERINI, 2011).

Os autores Quinderé, Jorge e Franco (2014), apresentaram resultados positivos em seus estudos realizados na Rede de Atenção Integral na Saúde Mental da cidade de Sobral-CE, que demonstraram que os serviços de saúde mental na localidade, dispõem de diferentes arranjos em rede de diversos dispositivos de cuidado que possibilitam a negociação de projetos terapêuticos menos medicalizantes. Pode-se notar a mobilidade dos trabalhadores na rede, circulando nos diversos campos de saúde, gerando o favorecimento das conexões e fluxos entre equipes na construção de linhas de cuidado, estes, tem por base a ideia de que todos são protagonistas no processo de cuidado e assim os movimentos são partilhados e articulados entre si.

\section{O agente comunitário de saúde e a saúde mental}

A seguir serão destacadas algumas pesquisas realizadas pelo Brasil relacionadas ao conhecimento dos ACS's acerca da saúde mental.

Os autores Silveira e Vieira (2009) verificaram em seus estudos três fatores que acabam por atenuar o desenvolvimento do trabalho dos ACS's, havia: a) a escassez de recursos humanos qualificados para 0 bom desenvolvimento do trabalho em saúde mental, principalmente aquelas conduzidas pela ESF. Alguns profissionais ainda hoje reproduzem o modelo biomédico que não priorizam o acolhimento e a escuta do sujeito, o que tem despotencializado as ações em saúde mental; b) as dificuldades na alcançabilidade dos usuários às ações e serviços de saúde mental; c) a necessidade de facilitação do processo de trabalho, já que observou-se a insuficiência de um trabalho coordenado entre os diversos profissionais de saúde mental e entre as demais equipes da unidade.

Correia, Barros e Colveiro (2011) evidenciaram que com o advento da Reforma Psiquiátrica, a priorização dos atendimentos e acompanhamento das pessoas com doença mental na comunidade implicaram no aumento da demanda desses pacientes nas unidades de saúde. Os esforços para assistência das pessoas em sofrimento psíquico na comunidade vêm crescendo, bem como a preocupação com as famílias, já que podem vivenciar problemas sociais, culturais, físicos e psicológicos. No entanto, observou-se que as ações de saúde mental desenvolvidas na atenção básica não apresentam uniformidade em sua execução.

Waidman, Costa e Paiano (2012), em seus estudos com agentes comunitários de saúde, observaram que nenhum dos profissionais entrevistados soube distinguir saúde de transtorno mental, alguns não tinham conhecimento suficiente para distinguir transtorno mental de problemas neurológicos, demonstraram sentimento de medo em relação à pessoa com transtorno mental e os descreveram como agressivos e agitados. Foi possível perceber também a falta de ações destinadas às pessoas com transtornos mentais, a demora nos serviços de referência e contrarreferência, a carência de profissionais e a falta de treinamento e capacitação para lidar com pessoas com transtornos mentais.

De acordo com Silva et al. (2014) existe um potencial de liderança e mobilização por parte dos ACS, porém suas percepções e ações estão calcadas no senso comum e isso acaba por restringir a efetividade de suas práticas que oscilam, entre falácias de que filantropia e assistência estão na base de sua atuação, e o descrédito da política levando em consideração a precariedade de condições de trabalho e a melhoria do serviço público de saúde.

Os estudos realizados por Silva-Junior et al. (2016) ratificou os resultados de Silveira e Vieira (2009) ressaltando a falta de qualificação dos ACS, e a visão distorcida sobre o portador de transtorno mental, que têm sido um grande bloqueio na assistência prestada por estes.

Em uma pesquisa realizada por Silva, Nóbrega e Oliveira (2018), sobre o conhecimento da equipe de enfermagem e agentes comunitários de saúde acerca do comportamento suicida, foi observado a falta de preparo dos profissionais que atuam nessa área para intervir, orientar e encaminhar aos serviços especializados de maneira adequada, assim como falta de comunicação e integração entre atenção básica e os centros de referência.

Ainda sobre Agentes Comunitários e sua atuação acerca das demandas do comportamento suicida, os autores Abreu, Eglê, Lima e Soares (2010) trouxeram em seus estudos que os ACS's relataram ter medo e receio para realizar abordagens com usuários com comportamento suicida, uma vez que se sentem insatisfeitos em relação as capacitações que recebem em saúde mental. Os resultados da pesquisa 
supracitada, corroborou com os estudos dos autores (CABRAL e ALBUQUERQUE, 2015).

\section{O comportamento suicida}

O comportamento suicida (SC) configura-se por três categorias: inicia com a ideação suicida que são pensamentos, ideias, planejamento e desejo de se matar, tentativa de suicídio e pode ser consumado com o ato em si (WERLANG; BORGES; FENSTERSEIFER, 2005).

Para a Organização Mundial da Saúde (2002), o suicídio pode ser conceituado como ato deliberado e levado a cabo por alguém que tem plena consciência de seu resultado final. No entanto, quando o suicida não consegue concluir o plano, passa a ser definido pela literatura como tentativa de suicídio. Então, considera-se que tanto a tentativa quanto o ato suicida em si foram motivados por ideações, que levaram o indivíduo a cogitar e planejar sua própria morte (CARDOSO et al., 2012).

O termo comportamento suicida se refere à ação de autoagressão, bem como variáveis relacionadas às tentativas de suicídio, com alta ou baixa letalidade, que ocorrem dentro de um contexto social, e trazem elementos que indicam a procura de ajuda. É necessário levar em consideração o plano suicida, o desejo de morrer contra o de viver, e as possibilidades de alguém descobrir o plano suicida para avaliar a intensidade do comportamento suicida (MANN, 2002 apud ABREU et al., 2010).

O autoextermínio é tido como fenômeno multidimensional, de repercussões inespecíficas, e representa, atualmente, importante problema de saúde pública, visto que, afeta não somente de maneira individual, como também coletiva. É relevante trazer também o crescimento em nível global, considerando o aumento das taxas de suicídio em $60 \%$ nos últimos 50 anos (BOTEGA, 2007).

O CS é um fenômeno multifatorial, de forma que o sofrimento é o que sustenta todos os fatores, e acaba levando o indivíduo à decisão de dar cabo a sua própria vida, como desenlace de uma continuação da ideia autodestrutiva (MARQUETTI, VILARUBIA e MILEK, 2014).

A ideação suicida se dá quando a pessoa passa a pensar e a se comportar pensando sempre em sua morte como melhor maneira, como forma mais equivalente e como maior recurso, passa a planejar e idear seu suicídio. "Tentativa de suicídio: Comportamento potencialmente autolesivo com consequências não fatais, acompanhado de evidências explicitas ou implícitas de que a pessoa tinha intenção de morrer" (QUEVEDO; SCHMITT; KAPCZINSKI, 2008, p.181).

Neste contexto, pudemos perceber que o suicídio é considerado como multifacetado, multifatorial e com várias interpretações. Brandão (2015) enfatiza que o suicídio pode ser uma resposta de esquiva ou fuga de situações aversivas. Logo, "o suicídio é um comportamento governado por regras, baseado na construção de consequências imaginárias".

Levando em consideração o comportamento suicida como um problema de saúde pública, foi que, no Brasil, em 2006, o Ministério da Saúde apresentou a Estratégia Nacional para Prevenção do Suicídio, com o intento de diminuir os óbitos e as tentativas, os danos associados e o impacto na família e também, nesse mesmo ano, foi lançado - Manual de Prevenção do Suicídio para Profissionais das Equipes de Saúde Mental, com o objetivo de detectar precocemente condições associadas ao fenômeno e realizar medidas de prevenção (BRASIL, 2006).

Sendo assim, do ponto de vista da prevenção do suicídio, as equipes de saúde que atuam no âmbito da atenção básica, por representarem o primeiro nível dos recursos de atenção à saúde, possuem elevado potencial para o desenvolvimento de estratégias e ações que propiciem a identificação e intervenção precoce em casos de risco de suicídio. As equipes da atenção básica possuem tal competência por estabelecerem o primeiro contato com a comunidade e por isso acabam criando vínculos que facilitam o oferecimento de ações que estejam de acordo com a necessidade dos usuários (SILVA, NÓBREGA e OLIVEIRA, 2018).

De maneira contextual, em Macapá, existe a Micro rede de atenção à crise suicida que se originou em maio de 2017 a partir do Ambulatório de Atenção à Crise Suicida (AMBACS), que é um projeto de pesquisa e extensão da Universidade Federal do Amapá (UNIFAP). A micro rede é composta de instituições governamentais municipais e federais, igrejas, instituição de ensino superior privada que estabeleceram convênio com o AMBACS, nas quais são realizados atendimentos de cunho psicológico individual em grupo (prevenção e pósvenção). Essas instituições estão distribuídas por diversos bairros em Macapá e Santana, no Amapá. Os atendimentos realizados têm seu fluxo iniciado no atendimento emergencial da crise suicida recebido no Hospital de Emergência (HE) e por demanda espontânea. Ao longo de 2018 e 2019 diversos esforços têm sido realizados para divulgar a micro rede nos âmbitos da educação, assistência social, justiça e saúde para facilitar o processo de acesso a serviço. Além disso, o AMBACS realizou parceria com o Ministério Púbico do Amapá para a produção de ações de escuta psicológica nos bairros de maior vulnerabilidade para o comportamento suicida bem como para a produção de um vídeo institucional enfatizando a prevenção do suicídio. 


\section{MÉTODOS}

Este estudo realizou-se através de uma pesquisa exploratório/descritiva de cunho qualitativo. No que se refere a este tipo de pesquisa, Gil (2014), narra que a exploratória tem a finalidade de proporcionar maior familiaridade com o problema (explicitá-lo), enquanto que descritiva visa descrever as características de determinadas populações ou fenômenos. Em se falando de abordagem qualitativa, Prodanov e Freitas (2013, p. 70) relatam que este tipo de pesquisa "considera que há uma relação dinâmica entre o mundo real e o sujeito", sendo que a interpretação dos elementos e a atribuição de significados são básicas nesse processo.

A pesquisa foi realizada na Unidade Básica de Saúde (UBS) Lélio Silva, localizada na cidade de Macapá-Ap, esta, lota duas equipes de ESF em suas dependências. A referida unidade visa atender a população do bairro do Buritizal e proximidades, com objetivo de prevenção e promoção de saúde no âmbito da Atenção Básica dirigida pelo SUS.

Participaram deste estudo seis Agentes Comunitários de Saúde (ACS's), integrantes de duas equipes de ESF que tinham um total de nove agentes em cada equipe, lotados na UBS Lélio Silva, porém, de acordo com os critérios de inclusão e exclusão apenas três agentes de cada equipe puderam participar. Os critérios de inclusão foram: estar ativo no período da coleta de dados, exercer suas atividades profissionais há mais de dois anos e ter assinado o Termo de Consentimento Livre e Esclarecido- TCLE. Foram excluídos deste estudo ACS's que estavam de férias, licenças ou qualquer situação legal relacionada à sua presença na equipe no período da coleta de dados, ACS's com menos de dois anos de atuação e que não assinaram o Termo de Consentimento Livre e Esclarecido- TCLE.

Como instrumento de pesquisa foi utilizada uma entrevista semiestruturada com 10 perguntas (APENDICE A). As perguntas procuravam informações sócio demográficas, o conhecimento do ACS acerca do seu papel e trabalho, o conhecimento do ACS sobre assistência em saúde mental, a relação do trabalho do ACS com a Rede de Atenção Psicossocial e o fazer no campo do comportamento suicida.

A presente pesquisa foi submetida à apreciação do Comitê de Ética Nacional (Plataforma Brasil) e somente após a aprovação sob o no. de Parecer 3.792.011 (ANEXO B) deste projeto, ela foi iniciada. A coleta de dados foi feita no período de 02 a 05 Janeiro de 2020, por meio da realização de entrevistas semiestruturadas gravadas em áudio.

Foi apresentado o Termo de Consentimento Livre e Esclarecido (TCLE) (APÊNDICE B) aos profissionais que pertencem a amostra da pesquisa, assim como o Termo de Anuência (ANEXO A) fornecido pela Secretaria de Saúde do Município de Macapá no próprio ambiente de trabalho, no intervalo da atividade laboral dos participantes.

Após a coleta de dados, as gravações de áudio das entrevistas foram transcritas na integra e foram analisadas, utilizando-se como técnica a análise de conteúdo. De acordo com Bardin (2011), a análise de conteúdo refere-se a técnicas que analisam as comunicações, com o propósito de obter, por meio sistemático de descrição das mensagens transmitidas, indicativos que possibilitem a inferência sobre conhecimentos relativos às mensagens produzidas $e$ recepcionadas.

Os dados foram analisados conforme categorias de registro construídas a partir dos relatos dos participantes para as questões contidas no questionário. As categorias de registro foram transformadas em categorias de análise conforme a seguir: O papel do ACS; A compreensão do ACS acerca da rede de saúde mental; A relação do trabalho do ACS com a RAPS; Ações vinculadas à assistência ao comportamento suicida e seus familiares.

A pesquisa foi desenvolvida de acordo com os parâmetros da Resolução do Conselho Nacional de Saúde (CNS) № 466/2012, que regulamenta pesquisas envolvendo seres humanos, sendo estes isentos de quaisquer danos materiais ou físicos.

\section{RESULTADOS E DISCUSSÃO}

Todos os participantes são do sexo feminino. A idade das profissionais envolvidas apresentou uma variação entre $34 \mathrm{e}$ 65 anos e uma média de 48 anos. Quatro participantes $(66,6 \%)$ com escolaridade nível médio e duas $(33,4 \%)$ com nível superior. As 6 participantes se declararam católicas. Sendo três participantes (50\%) solteiras, duas $(33,4 \%)$ divorciadas e uma $(16,6 \%)$ casada.

A seguir, os resultados serão apresentados obedecendo as categorias de análise: O papel do ACS; A compreensão do ACS acerca da saúde mental; A relação do trabalho do ACS com a RAPS; Ações vinculadas à assistência ao comportamento suicida e seus familiares.

\section{Qual o papel do agente comunitário de saúde?}

A respeito do questionamento feito aos ACSs em relação ao papel e trabalho destes, seis participantes responderam que consiste em prevenir doenças, destes, três acrescentaram sobre o papel de orientar as famílias, apenas um relatou o papel de encaminhar, e um também acrescentou o papel do ACS como aquele que faz a ligação 
entre a comunidade e o serviço de saúde.

A seguir serão apresentados no Quadro 1, exemplos dos relatos que caracterizam as ênfases dadas pelos ACS's para descrever suas atribuições.

Uma das principais atribuições do ACS na ESF é coletar informações referentes às necessidades de saúde da população, identificar usuários com problemas e encaminhálos a unidade de saúde. E por ser integrante da comunidade, o ACS torna-se um mediador e facilitador na relação entre o serviço de saúde e seu usuário, estabelecendo uma comunicação interpessoal efetiva (WAIDMAN; COSTA; PAIANO, 2012).

Entendemos que as informações transmitidas pelos ACS's está de acordo com o que a literatura indica e ainda segundo a Portaria $N^{\circ} 2.436$, de 21 de Setembro de 2017, que trata da revisão de diretrizes da Política Nacional da Atenção Básica e atribui aos a Agentes Comunitários de Saúde o dever de desenvolver atividades de promoção da saúde, de prevenção de doenças e agravos, em especial aqueles mais prevalentes no território, e de vigilância em saúde, por meio de visitas domiciliares regulares e de ações educativas individuais e coletivas, bem como identificar casos suspeitos de doenças e agravos, encaminhar os usuários para a unidade de saúde de referência, registrar e comunicar o fato à autoridade de saúde responsável pelo território.

É principalmente por meio desse contato que o profissional identifica situações de sofrimento e consegue melhorar suas ações por intermédio da escuta e dos encaminhamentos. Esse elo beneficia a criação de redes onde o ACS consegue atingir seu objetivo com a sua atuação e o usuário com atendimento para a sua demanda.

\section{Compreensão do ACS'S acerca da Assistência em Saúde Mental}

A compreensão que os ACS's relataram acerca da assistência em saúde mental demonstrou-se diversificada. Uma participante relacionou a assistência às pessoas em uso abusivo de álcool e drogas, duas participantes relacionaram a distúrbio ou transtorno mental, uma participante relacionou a problemas neurológicos, uma não soube responder e apenas uma respondeu que é o equilíbrio das emoções e bem-estar.

A seguir serão apresentados no Quadro 2, exemplos dos relatos que caracterizam o conhecimento do ACS acerca da assistência em saúde mental.

Observamos que a participante $\mathrm{A} 1$ enfatizou o tipo de assistência fornecida às pessoas em uso abusivo de álcool e outras drogas, prevista na Lei Federal no 10.216 de 2001, conhecida como lei antimanicomial que propõe a estruturação e fortalecimento de uma rede de assistência centrada na atenção comunitária, com ênfase na reabilitação e reinserção social dos usuários (SANTOS e FERLA, 2017).

Nos relatos das participantes $A 2, A 3$ e $A 6$ pode-se evidenciar ênfase às dimensões saúde e doença, parece confundirem-se ou relacionam a quadros de adoecimento, acompanhamento clínico especializado e uso de medicação. Essa relação acaba por centrar o trabalho no modelo biomédico não visando ações preventivas, o que corrobora com os resultados dos autores Silveira e Vieira (2009), que apresentou a escassez de recursos humanos qualificados para o bom desenvolvimento do trabalho em saúde mental,

Quadro 1. Caracterização do papel e do trabalho do ACS na percepção destes.

\begin{tabular}{|l|l|}
\hline ACS & RESPOSTAS \\
\hline A1 & Prevenir doenças, levar informações, levar profissionais e controle de doenças. \\
\hline A2 & Orientar para prevenir doenças. \\
\hline A3 & Prevenção e identificação de doenças para encaminhar para a coordenação. \\
\hline A4 & Acompanhamento de famílias visando orientar para prevenção de doenças. \\
\hline A5 & $\begin{array}{l}\text { Fazer a ligação entre comunidade e UBS para orientação das famílias acerca de } \\
\text { prevenção de doenças }\end{array}$ \\
\hline A6 & Trabalha com prevenção e orientação das famílias. \\
\hline
\end{tabular}

Quadro 2. Conhecimento do ACS acerca da assistência em saúde mental

\begin{tabular}{|l|l|}
\hline ACS & RESPOSTA \\
\hline A1 & É a assistência às pessoas em uso abusivo de álcool e outras drogas. \\
\hline A2 & Saúde mental é você não estar bem da cabeça, estar com algum distúrbio. \\
\hline A3 & Problema neurológico, deficiência, agressão física, psíquica ou maus tratos. \\
\hline A4 & Não sei. \\
\hline A5 & É o equilíbrio das emoções, estar bem com você e com as pessoas do seu convívio. \\
\hline A6 & Pessoas com transtornos e doenças mentais que precisam de acompanhamento. \\
\hline
\end{tabular}


principalmente aquelas conduzidas pelo PSF e da insuficiência de um trabalho coordenado entre os diversos profissionais de saúde mental e entre as demais equipes da unidade. A participante A4 não soube responder e a participante A5 fez uma associação breve e discreta relacionada ao equilíbrio de emoções e bem-estar, talvez enfocando o objetivo assistencial em saúde mental.

Pode-se perceber a falta de uma definição mais clara e objetiva acerca da indagação feita aos profissionais, logo entendemos que estes não possuem um conhecimento sólido a respeito da assistência em saúde mental, o que vem a reforçar as nossas hipóteses de que o fortalecimento da assistência a saúde mental está atrelado ao conhecimento que os ACS apresentam acerca da política de assistência em saúde mental e o papel que eles desenvolvem dentro desta.

\section{A Relação do Trabalho dos ACS's com a Rede de Saúde Mental}

No que diz respeito a relação do trabalho do ACS com a Rede de Saúde Mental as participantes A1 e A6 responderam que os ACS's em conjunto ás equipe de ESF tem apoio do NASF-AB, o qual, tem por objetivo apoiar a Estratégia de Saúde da Família (ESF) e outras modalidades de equipes de atenção básica, ampliar o escopo de atuação desses grupos, proporcionar uma retaguarda especializada nas ações de saúde e de aumentar a resolutividade do cuidado em saúde na atenção básica (BRASIL,2014). Além disso, o modo de organizar o processo de trabalho do NASF-AB é norteado, principalmente, pela lógica do apoio matricial, clínica ampliada, cogestão e por ferramentas que subsidiem o trabalho como, por exemplo, o Projeto de Saúde no Território e o Projeto Terapêutico Singular (BRASIL,2014).

É importante ressaltar a fala da participante $A 1$ no que se refere a falta de feedback em relação aos atendimentos dos pacientes, a mesma relatou que encaminha para o psicólogo do NASF-AB, porém não tem conhecimento dos procedimentos após o encaminhamento. Os dados encontrados são corroborados pelo estudo de Silva, Nóbrega e Oliveira (2018), que enfatiza a falta de comunicação e integração entre atenção básica e Centros de Referência. Isto posto, podemos notar que a articulação e integração entre os profissionais de saúde mental que prestam atendimento na unidade e os técnicos de saúde mental envolvidos com o trabalho na ESF, NASF-AB, podem estar pouco ajustadas a política de saúde mental com vistas a boa articulação em relação a integração e matriciamento.

A participante A2 mencionou os psicólogos da UBS. A UBS também faz parte da RAPS e Atenção Básica, e lota em seus espaços físicos as equipes de ESF, bem como dar apoio. A participante $A 3$ não fez menção a nenhum outro órgão da Rede de Assistência, as participantes A4 e A5 não souberam responder. Sendo assim, a metade das participantes do estudo não responderam adequadamente a indagação. Conforme quadro 3.

A seguir serão apresentados no Quadro 3, exemplos dos relatos dos ACS que caracterizam a relação do trabalho do ACS com a Saúde Mental.

Foi perceptível a falta de conhecimento dos ACS's da possibilidade de trabalhar junto aos CAPS, como sugere Schneider et al., (2014), estes citaram apenas a própria equipe, UBS e NASF-AB, parecendo desconhecer outros dispositivos da RAPS que dão suporte a Atenção Básica, o que despotencializa as ações.

Corroboramos também com Silveira e Vieira (2009) quando trazem sobre insuficiência de um trabalho coordenado entre os diversos profissionais de saúde mental e entre as demais equipes da unidade, o que vem a enfraquecer a política de saúde mental. É importante levar em consideração também que a metade das participantes desconhecem totalmente a Rede de Atenção Psicossocial.

\section{Ações vinculadas d̀ Assistência Comportamento Suicida e seus Familiares}

ao

No que se refere a questão do trabalho do ACS com pessoas que tentaram suicídio, se autolesionam e seus familiares, as participantes A1, A2, A4 e A5 evidenciaram adequadamente a conduta de encaminhamento, uma vez que, os serviços da ESF requer uma adequação da lógica do

Quadro 3. A Relação do Trabalho do ACS com a Saúde Mental

\begin{tabular}{|l|l|}
\hline ACS & RESPOSTA \\
\hline A1 & Temos o NASF, mas não temos Feedback dos atendimentos e procedimentos. \\
\hline A2 & Temos os psicólogos na unidade, encaminhamos para eles. \\
\hline A3 & Encaminhamos para o médico, que encaminha para as demais especialidades. \\
\hline A4 & Não sei. \\
\hline A5 & Não sei. \\
\hline A6 & Trabalhamos em conjunto com a UBS e NASF \\
\hline
\end{tabular}


SUS para os programas de Atenção à Saúde Mental, com vistas a identificar rapidamente a sintomatologia, diagnosticar, estabelecer a terapêutica e proceder ao devido encaminhamento, promovendo a instrumentalização teórica e prática dos profissionais envolvidos como uma das estratégias dos programas de sistema de saúde (SILVA; DALMASENO, 2002). Porém, o fluxo de encaminhamento não deixa claro se a Micro rede de Atenção à Crise Suicida é inserida.

Três participantes citaram que conversam e escutam os familiares, enfatizando adequadamente a necessidade da fala com acolhimento e empatia. Lacerda (2010), descreve em seus estudos a importância dos vínculos que se manifestam oriundos de conversas e contato entre os elementos. É por meio dessa relação que o ACS identifica as demandas e consegue potencializar suas ações.

A seguir serão apresentados no Quadro 4, exemplos dos relatos dos ACS que caracterizam como lidam com pessoas que tentaram suicídio, se autolesionam e com seus familiares.

Pode-se perceber que os ACS's realizam a conduta de encaminhamento, que é inerente as suas atribuições, mas não deixaram claro se a Micro rede está inserida nesses encaminhamentos, conversam e escutam os familiares enfatizando adequadamente a necessidade da fala com acolhimento e empatia, por outro lado também há a necessidade de ações além do encaminhamento, que acatem o sofrimento das famílias, uma vez que, estes são importantes no tratamento e na recuperação das pessoas envolvidas no comportamento suicida.

\section{CONCLUSÕES}

O objetivo desta pesquisa foi analisar o conhecimento que os Agentes Comunitários de Saúde (ACS's) apresentam acerca da assistência em saúde mental e comportamento suicida no Município de Macapá, Amapá, tendo por objetivos específicos identificar as atribuições dos ACS's no campo da promoção de saúde mental, caracterizar a compreensão que os ACS's têm acerca da assistência em saúde mental e compreender o conhecimento que os ACS's têm acerca da assistência ao comportamento suicida. Através da metodologia proposta, conseguimos alcançar os objetivos geral e específicos.

Por meio deste estudo foi possível entender a importância da ESF na política de saúde mental, e que o ACS desempenha um importante papel para o bom andamento das ações, levando em consideração que este, estrategicamente, é o profissional que tem o primeiro e maior contato com a comunidade e pode identificar problemas antes da equipe de saúde.

Com este estudo conseguimos perceber que a maioria dos ACS's define o seu papel satisfatoriamente quanto a prevenção de doenças, coleta de informações, identificação de demanda e encaminhamento, bem como, promover saúde. Seu engajamento no território tem um importante potencial de escuta, o que facilita o desenvolvimento de ações voltadas para demandas especificas.

Isto posto, é importante discutir acerca da reprodução do modelo biomédico, centrado apenas no tratamento e na medicalização de doenças, sem promover ações voltadas para a prevenção no campo da saúde mental e isso acaba divergindo com o modelo de cuidado pensado pelo ESF e dispositivos de cuidado em saúde mental. Foi perceptível também a falta de integração dos dispositivos da RAPS, dificultando assim o bom andamento dos atendimentos e a desburocratização dos serviços.

É importante também realçar a necessidade de constantes atualizações relacionadas a capacitação destes profissionais no campo da saúde mental.

Este estudo torna-se importante para a consolidação de práticas voltadas para um cuidado adequado às pessoas, possibilitando a integração de forma positiva dos dispositivos de assistência em saúde mental, tanto no fortalecimento da necessidade de formação continuada quanto no fazer diário em busca de um cuidado mais humanizado.

A limitação deste estudo é o tamanho da amostra, sendo assim, os resultados encontrados não podem ser generalizados, afinal, esta pesquisa é o ponto de partida para novas investigações no campo da saúde mental na localidade supracitada.

\section{REFERÊNCIAS}

ABREU, K. P. de; LIMA, M. A. D; EGLÊ, K; SOARES, J. dos S. S. Comportamento suicida: fatores de risco e intervenções preventivas. Revista Eletrônica de Enfermagem, 12(1), 195-200, 2010.

AMARANTE, P; GIOVANELLA, L. O enfoque estratégico do planejamento em saúde mental. In: Amarante $P$, organizador. Psiquiatria social e reforma psiquiátrica. Rio de Janeiro: Fiocruz; 1998. p. 113-48.

BARBOSA, L. de A; DANTAS, T. M. Percepção dos agentes comunitários de saúde sobre saúde da família. Saúde $\&$ Transformação Social/Health \& Social Change. Florianópolis, Santa Catarina. 2013; 4(1):73-81.

BARDIN, L. Análise do Conteúdo. São Paulo: Edições 70, 2011.

BOTEGA, N. J. Suicídio: Saindo da sombra em direção a um plano nacional de prevenção. [editorial] Rev Bras 
Psiquiatr. 2007;29(1):7-8.

BRANDÃO, W. L. de O. Comportamento suicida: sociedade, assistência e relações comportamentais. Programa de Pós- Graduação em Teoria e Pesquisa do Comportamento. Belém, p.162, 2015.

BRASIL. Portaria n. 336/2002, de 19 de fev. 2002. Brasília: Ministério da Saúde. Disponível em: Acesso em: 25 de Maio de 2019.

BRASIL. Ministério da Saúde. Secretaria de Atenção a Saúde. Departamento de Ações Programáticas Estratégicas. Saúde mental no SUS: os centros de atenção psicossocial. Brasília: Ministério da Saúde, 2004a.

BRASIL. Ministério da saúde. Coordenação de saúde Mental. 2005, Reforma psiquiátrica e política de saúde mental no Brasil. Brasília.

BRASIL. Portaria n.1.876, de 14 de agosto de 2006. Define diretrizes nacionais de prevenção ao suicídio. Diário Oficial da União 2006; 15 ago.

BRASIL. Decreto no 7.508, de 28 de junho de 2011. Regulamenta a lei no 8.080, de 19 de setembro de 1990, para dispor sobre a organização do Sistema Único de Saúde - SUS, o planejamento da saúde, a assistência à saúde e a articulação interfederativa, e dá outras providências à Rede de Atenção à Saúde. Disponível em: http://www.planalto.gov.br/ccivil 03/_ato2011-

2014/2011/decreto/D7508.htm. Acesso em: 15 de Maio de 2019.

BRASIL. Ministério da Saúde. Rede de Atenção Psicossocial. Portaria no 3.088, 23 de dezembro de 2011. Brasília: Ministério da Saúde. 23 de dez. 2011. Disponível em: http://bvsms.saude.gov.br/bvs/saudelegis/gm/2011/prt3 08823122011 rep.html. Acesso: 03 jan.2020.

BRASIL. Ministério da Saúde. Núcleo de Apoio à Saúde da Família: ferramentas para gestão e para o trabalho cotidiano. v. 1. Brasília: Ministério da Saúde, 2014. 118 p. (Cadernos de Atenção Básica, n. 39)

CABRAL, T. M. N; ALBUQUERQUE, P. C. Saúde mental sob a ótica de Agentes Comunitários de Saúde: a percepção de quem cuida. Saúde debate, Rio de Janeiro, v. 39, n. 104, p. 159-171, Mar. 2015 . Available from <http://www.scielo.br/scielo.php?script=sci_arttext\&pid =S0103-11042015000100159\&lng=en \&nrm=iso>. Acesso em 16 Jun. 2020. http://dx.doi.org/10.1590/0103110420151040415.

CAMPOS, G. W. de S; DOMITTI, A. C. Apoio matricial e equipe de referência: uma metodologia para gestão do trabalho interdisciplinar em saúde. Cad. Saúde Pública, Rio de Janeiro, v. 23, n. 2, p. 399-407, Feb. 2007 . Available from

<http://www.scielo.br/scielo.php?script=sci_arttext\&pid =S0102-311X2007000200016\&lng=en\&nrm=iso>. Acesso em 05 Jun. 2019. http://dx.doi.org/10.1590/S0102311X2007000200016.

CAMPOS, G. W. S. Produção de conhecimento, avaliação de políticas públicas em saúde mental: notas reflexivas. In: CAMPOS, R.O. et al. Pesquisa avaliativa em Saúde Mental: desenho participativo e efeitos da narratividade. São Paulo: Aderaldo \& Rothschild, 2008. p. 97-102.

CAMPOS, G. W. S. Equipes de referência e apoio especializado matricial: um ensaio sobre a reorganização do trabalho em saúde. Ciênc. saúde coletiva, Rio de Janeiro, v. 4, n. 2, p. 393-403, 1999 . Available from <http://www.scielo.br/scielo.php?script=sci_arttext\&pid $=\mathrm{S} 1413-81231999000200013 \& \operatorname{lng}=\mathrm{en} \& \mathrm{nrm}=\mathrm{iso}>$.

Acesso em:

06 Jun. 2019. http://dx.doi.org/10.1590/S141381231999000200013.

CARDOSO, H. F; BAPTISTA, M. N; VENTURA, C. D; BRANDÃO, E. M; PADOVAN, F. D; GOMES, M. A. Suicídio no Brasil e América Latina: revisão bibliométrica na base de dados Redalycs. Diaphora, [S.I.], v. 12, n. 2, p. 42-48, nov. 2014. ISSN 2238-9709. Disponível em: <http://www.sprgs.org.br/diaphora/ojs/index.php/diaph ora/article/view/69/69>. Acesso em: 12 de Jun. 2019.

CARDOSO, A. V. M; REINALDO, A. M. S; CAMPOS, L. F. Conhecimento dos agentes comunitários de saúde sobre transtorno mental e de comportamento, em uma cidade de Minas Gerais. Cogitare enferm, 2008; 13(2): 235-43.

CECILIO, Saúde Públ., Rio de Janeiro, 13(3):469-478, jul-set, 1997.

CHIAVERINI, D. H. (Org.). Guia prático de matriciamento em saúde mental. Brasília (DF): Ministério da Saúde/Centro de Estudo e Pesquisa em Saúde Coletiva, 2011. CONSOLI, G. L; HIRDES, A; COSTA, J. S. D. Saúde mental nos municípios do Alto Uruguai, RS, Brasil: um diagnóstico da reforma psiquiátrica. Ciênc. saúde coletiva, Rio de Janeiro, v. 14, n. 1, p. 117128, fev. 2009 . Disponível em <http://www.scielo.br/scielo.php?script=sci_arttext\&pid $=$ S1413-81232009000100017\&lng=pt\&nrm=iso $>$. Acesso em 15 Dez. 2019. http://dx.doi.org/10.1590/S141381232009000100017.

CORREIA, V. R; BARROS, S; COLVERO, L. A. C. Saúde mental na atenção básica: prática da equipe de saúde da família. Revista da Escola de Enfermagem da Universidade de São Paulo, São Paulo, v. 45; n. 6; p. 1501-1506; 2011. Disponível em

http://www.scielo.br/scielo.php?script=sci arttext\&pid= s0080-62342011000600032\&lng=en\&nrm=iso> Acesso em 12 de Jun de 2019. http://dx.doi.org/10.1590/S008062342011000600032.

FORTES, P. A. C; SPINETTI, S. R. O agente comunitário de 
saúde e a privacidade das informações dos usuários. Cadernos de Saúde Pública, Rio de Janeiro, v. 20, n. 5, 2004, p. 1328-1333.

GIL, A. C. Métodos e técnicas de pesquisa social. São Paulo: Atlas, 2014.

LACERDA, A. Redes de apoio social no Sistema da Dádiva: um novo olhar sobre a integralidade do cuidado no cotidiano de trabalho do agente comunitário de saúde. 2010. $204 \mathrm{f}$. Tese (Doutorado em Saúde Pública) - Escola Nacional de Saúde Pública Sergio Arouca, Fundação Oswaldo Cruz, Rio de Janeiro, 2010.

LOPES, A. M. P; SOUZA, F. Agentes comunitários de saúde na construção da reforma psiquiátrica comunitária. Cadernos Brasileiros de Saúde Mental, ISSN 1964-2147, Florionopólis, V. 2, n 4-5, p28- 46; 2010.

MARQUETTI, F. C; VILARUBIA, G. V; MILEK, G. Percurso suicida: observação e análise de alterações no cotidiano do indivíduo com tentativas de suicídio. Revista de Terapia Ocupacional da Universidade de São Paulo. São Paulo. v. 25, n. 1, p. 18-26. 2014. Disponível em: < http://www.revistas.usp.br/rto/article/view/64664> Acesso: 08/09/2018.

MINISTÉRIO DA SAÚDE. Guia Prático de Matriciamento em Saúde. Ano:2011. Disponível em: <www.unisite.ms.gov.br>. Acesso em: 11 de ago. de 2013.

NUNES, L. I. Mídia e suicídio: prevenção e posvenção na era digital. 2018. 11-80. Trabalho de Conclusão de Curso (Monografia) Bacharel em Psicologia, Instituto Macapaense do Melhor Ensino Superior, Macapá-Ap, 2018.

NUNES, M. O; TRAD, L. B; ALMEIDA, B. A; HOMEM, C. R; MELO, M. C. I. C. O agente comunitário de saúde: construção da identidade desse personagem híbrido e polifônico. Cad. Saúde Pública, Rio de Janeiro , v. 18, n. 6, p. 1639-1646, Dec. 2002 . Available from $<$ http://www.scielo.br/scielo.php?script=sci_arttext\&.pid =S0102-311X2002000600018\&lng=en \&nrm=iso >.

\section{Accesso}

em 15 Maio. 2019. http://dx.doi.org/10.1590/S0102311X2002000600018.

ORGANIZAÇÃO MUNDIAL DA SAÚDE. Relatório mundial sobre violência e saúde. Genebra: OMS; 2002.

PRODANOV, C. C; FREITAS, E. C. Metodologia do trabalho científico: métodos e técnicas da pesquisa e do trabalho acadêmico. Novo Hamburgo: Feevale, 2013.

QUEVEDO, J; SCHMITT, R; KAPCZINSKI, F. Emergências Psiquiátricas. 2a ed. Porto Alegre: Artmed; 2008.

QUINDERE, P. H. D; JORGE, M. S. B; FRANCO, T. B. Rede de Atenção Psicossocial: qual o lugar da saúde mental?. Physis, Rio de Janeiro, v. 24, n. 1, p. 253-
271, mar. 2014 . Disponível em <http://www.scielo.br/scielo.php?script=sci_arttext\&pid =S0103-3312014000100253\&lng=pt\&nrm=iso>. Acesso em 15 Maio 2019. http://dx.doi.org/10.1590/S010373312014000100014.

SANTOS, F. F.; FERLA, A. A. Mental health and primary care in alcohol and drug users care. Interface (Botucatu). 2017; 21(63):833-44. Disponível em < http://www.scielo.br/pdf/icse/v21n63/1807-5762-icse1807-576220160270.pdf> Acesso em 08 de Novembro de 2019.

SCHNEIDER, D. R;. CERUTTI, M. G; MARTINS, M. T; NIEWEGLOWSKI, V. H. A atuação do psicólogo no centro de atenção psicossocial voltado para álcool e outras drogas (CAPSad): os desafios da construção de uma clinica ampliada. R. Eletr. de Extensão, Florianópolis, v 11, n17, p.101-113, 2014.

SILVA, J; DALMASO, A. W. Agente comunitário de saúde: o ser, o saber, o fazer. Rio de Janeiro: Fiocruz, 2002.

SILVA, C. R. C. S; MENDES, R; MORAES, R. C. P; ANHAS, D. M; ROSA, K. R. M. Participação social e a potência do agente comunitário de saúde. Psicol. Soc., Belo Horizonte, v. 26, n. spe2, p. 113-123, 2014 . Available from $<$ http://www.scielo.br/scielo.php?script=sci_arttext\&pid =S0102-71822014000600012\&lng=en\&nrm=iso>. Acesso em 16 Jun. 2019. http://dx.doi.org/10.1590/S010271822014000600012 .

SILVA, P. F; NÓBREGA, M. P. S. S; OLIVEIRA, E de. Conhecimento da equipe de enfermagem e agentes comunitários sobre o comportamento suicida. Revista de Enfermagem da UFPE 2018; 12(1):112-117.

SILVA-JUNIOR, R. F; ARAÚJO, D. F; SILVA, N. K. F; TORRES, S. A. S; DINIZ, H. J. C; TORRES, J. D. R.V. O papel do agente comunitário de saúde no atendimento ao portador de transtorno mental. Revista Eletrônica Acervo Saúde, 2016. Vol. 8 (1), 841-845.

SILVEIRA, Daniele Pinto da; VIEIRA, Ana Luiza Stiebler. Saúde mental e atenção básica em saúde: análise de uma experiência no nível local. Ciência \& Saúde Coletiva, 2009, 14(1), 139-148.

TENORIO, F. A reforma psiquiátrica brasileira, da década de 1980 aos dias atuais: história e conceitos. Hist. cienc. saude-Manguinhos, Rio de Janeiro , v. 9, n. 1, p. 2559, Apr. 2002 . Available from <http://www.scielo.br/scielo.php?script=sci_arttext\&pid =S0104-59702002000100003\&lng=en\&nrm=iso>. Acesso on 03 Fev. 2019. http://dx.doi.org/10.1590/S010459702002000100003

TRAPÉ, T. L; CAMPOS, R. O. Modelo de atenção à saúde mental do Brasil: análise do financiamento, governança e mecanismos de avaliação. Rev Saude Publica. 
2017;51:19.

VIEIRA FILHO, N. G; NÓBREGA, S. M. A atenção psicossocial em saúde mental: contribuição teórica para o trabalho terapêutico em rede social. Rev. Estudos de Psicologia. Natal, v. 9, n. 2, p. 373-379, 2004.

VIEIRA, A. M. B; AlbuQUerque, E. G; PEREIRA, L. A; ANDRADE, I. S. Percepção dos Agentes Comunitários de Saúde acerca do Acompanhamento aos Usuários de Saúde Mental. SANARE, Sobral - v.16. n. 01, p.24-312017.

WAIDMAN, M. A. P; COSTA, B; PAIANO, M. Percepções e atuação do Agente Comunitário de Saúde em saúde mental. Rev. esc. enferm. USP, São Paulo , v. 46, n. 5, p. 1170-1177, out. 2012 . Disponível em <http://www.scielo.br/scielo.php?script=sci_arttext\&pid =S0080-62342012000500019\&lng=pt\&nrm=iso>. Acesso em 09 Maio. 2019. http://dx.doi.org/10.1590/S008062342012000500019.

WERLANG, B. S. G; BORGES, V. R; FENSTERSEIFER, L. (2005). Fatores de risco ou proteção para a presença de ideação suicida na adolescência. Revista Interamericana de Psicologia, 2005, 39(2), 259-266.

Submissão: $11 / 03 / 2020$

Aprovado para publicação: 22/09/2020 